\title{
Allelopathic effect of Chitosan Fiber on the growth of Microcystis aeruginosa
}

\author{
Jingguo Cao ${ }^{1,2, *}$, Hongyan Zhao ${ }^{1}$, Zezhang Dong ${ }^{1}$, Zongzheng Yang ${ }^{1, *}$ \\ ${ }^{1}$ College of Chemical Engineering and Material, Tianjin University of Science \&Technology, TEDA, Tianjin 300457, PR China \\ ${ }^{2}$ Tianjin Key Laboratory of Marine Resources and Chemistry, (Tianjin University of Science \& Technology), Tianjin, 300457
}

\begin{abstract}
Cyanobacterial bloom elimination has become an urgent concern in aquatic environment protection. Allelopathic technology, however, is one of the potential effective approaches because of its exclusive and ecological safety protection. The potential application of chitosan fiber in the inhibition of Microcystis aeruginosa was explored for the first time. Results showed that the cell growth of $M$. aeruginosa was inhibited effectively by chitosan fibers. After 12 days of treatment, the inhibition rate (IR) of algal cells was above $78.9 \%$; those of chlorophyll a and carotenoids in algal cells were $86.9 \%$ and $93.1 \%$, respectively; those of phycocyanin, allophycocyanin and phycoerythrin in phycobiliproteins were all approximately $100 \%$; those of soluble proteins and algal toxins were $88 \%$ and $100 \%$, respectively; and those of superoxide dismutase and catalase activities were $68 \%$ and $74 \%$, respectively. In addition, chitosan fiber effectively destroyed the structure of algal cells involved in the photosynthetic process, thereby destroying the metabolic activity of algal cells. This study provides a novel method utilizing a new allelopathic material.
\end{abstract}

\section{Introduction}

Cyanobacteria are the first photoautotrophic prokaryotes on the earth that performs photosynthesis [1]. In eutrophic lakes and freshwater bodies such as reservoirs and rivers, cyanobacterial growth increases to a large scale when the water temperature $\left(>25{ }^{\circ} \mathrm{C}\right)$, light, nutrients, nitrogen and phosphorus $(\mathrm{N}, \mathrm{P})$ ratio are suitable. Cyanobacterial growth is intensified by suitable hydrological conditions accompanied by low pressure, weak precipitation, and low wind speed. Algal groups float, drift, and aggregate in the water body visible to human, whereas green-floating foam or algal pulp can be found on the water surface.

With the increased eutrophication of global water bodies, cyanobacterial blooms have become a pollutant issue for water environment and human health. After the breakout of cyanobacterial blooms, water transparency is substantially reduced, the dissolved oxygen in the water is consumed, and the $\mathrm{pH}$ of the water body is remarkably increased. These drastic changes in the water environment will lead to the death of a large number of aquatic organisms, corrupted and odorous water quality, and destruction of the structure and diversity of the water ecosystem [2]. Secondary toxic metabolites such as cyanobacterial toxins may also be released by certain cyanobacteria into the water bodies [3]. Especially in the late stage of cyanobacterial bloom, a large number of cyanobacterial cells ruptured, and cyanobacterial toxins such as microcystins (MCs) are released into the water body, leading to further deterioration of water quality that becomes harmful to the safety of animal and human health. The use of algicides, including various chemical or synthetic agents such as diuron [4], copper sulfate [5], and endothall [6], is effective within a short period after application but might be dangerous because of the persistence, lack of selectivity, and minimal safety margins.

Natural plant agents (allelochemicals) for inhibiting cyanobacterial blooms have recently received attention due to their specificity and biodegradability [7]. Isolation of allelopathic compounds from aquatic plants has been widely studied [8-9], but the algicidal properties of marine life has received less attention.

Chitosan fiber, also known as polyglucosamine, is a polymer obtained by linking a plurality of glucosamine monomers through $\beta$-1,4-glycosidic bonds, which are formed from molecular linear polysaccharide by partially or completely removing acetyl from chitin [10]. Its monomeric formula is $\left(\mathrm{C}_{6} \mathrm{H}_{11} \mathrm{NO}_{4}\right) \mathrm{N}$. Chitosan fiber is usually isolated from shrimp and crab shell and is rich in storage, renewable, and ecologically friendly. Chitosan fiber is the only kind of natural alkaline polysaccharide discovered thus far and can be widely used because of its non-toxicity to human, good biocompatibility, biodegradability, and broad-spectrum antibacterial properties. This material has received increased attention and was used for related research. Studies on the antibacterial properties of chitosan fibers have focused on bacteria and fungus [11]. However, no report is

*Correspondence: cjg@tust.edu.cn yzz320@tust.edu.cn 
available on the use of chitosan fibers to inhibit cyanobacteria.

In this work, chitosan fiber was used as an algaecide inhibitor. The effects on cell structure, photosynthetic system, protein synthesis, and algal toxin were studied. Chlorophyll a, carotenoid, phycobiliprotein, and lipid peroxidation index were measured. The concentrations of malondialdehyde (MDA), superoxide dismutase (SOD), catalase (CAT) activity and algal toxin release, were analyzed to reveal the mechanism of chitosan fiber inhibition of cyanobacteria.

\section{Materials and methods}

\subsection{M. aeruginosa and chitosan fiber}

The cultivation of algae is reported by Duan, $\mathrm{S} \mathrm{H}$ [12]. The chitosan fiber used in this experiment was provided by Tianjin Zhongsheng Biological Engineering Co., Ltd., China.

\subsection{Experimental}

In brief, $10 \mathrm{~g}$ of chitosan fiber was added to $1 \mathrm{~L}$ of $\mathrm{M}$. aeruginosa culture medium. The initial density and OD(optical density) value of the algal liquid was recorded and measured every 3 days. Determination methods for chlorophyll a, carotenoid, phycobiliprotein (Phycoerythrin(PE), Allophycocyanin,(APC) and Phycocyanin (PC)), and microcystins (LR) are described in the literature [13-14]. Soluble protein was measured according to Bradford method [15].

\subsection{OD value and Scanning Electron Microscope (SEM)}

SEM was used for figure observation of the surface of Microcystis after chitosan fiber treatment. The cells were processed according to a previous method and observed through SEM (SU1510) operating at a voltage of $30 \mathrm{kV}$ [16].

\subsection{Determination of cell membrane permeability}

In brief, $15 \mathrm{~mL}$ of algae solution was placed in a centrifuge tube and centrifuged at $7000 \mathrm{r} / \mathrm{min}$ for $10 \mathrm{~min}$. The supernatant was investigated for the following parameters. Non-electrolyte extravasation amount was determined by the ultraviolet absorption method using the OD264 value. The supernatant was then filtered through a $0.2 \mu \mathrm{m}$ microfiltration membrane, and its $\mathrm{K}+$ concentration was measured by ion chromatography (PIC-10, Qingdao Puren Instrument Co., Ltd.).

\subsection{MDA, SOD and CAT}

MDA content was determined by thiobarbital acid colorimetry [17]. SOD and CAT activities were determined with a kit purchased from China Solarbio.

\subsection{Data analysis}

Three parallel samples were set up during all the experiments, and the origin 9.0 software was used for illustration. Inhibition rate (IR) was calculated according to The formula as follows:

$$
I R=\left(1-E_{n} / C_{n}\right) \times 100 \%
$$

where En and $\mathrm{Cn}$ represent the data from experimental group and control group, respectively.

\section{Results and discussion}

\subsection{Inhibition effect on the growth of $M$. aeruginosa}

Fig. 1A shows that chitosan fiber has a significant inhibitory effect on $\mathrm{M}$. aeruginosa growth. The OD value in the control group was increased from 0.978 to 1.636 , whereas that in the treatment group was reduced from 0.978 to 0.348 . Compared with the control group, the M. aeruginosa cell concentration in the chitosan fiber treatment group was significantly reduced on the 6th day of the experiment, and the IR reached $55 \%$. With the increase in experimental time, the IR reached $79 \%$ on the 12 th day.
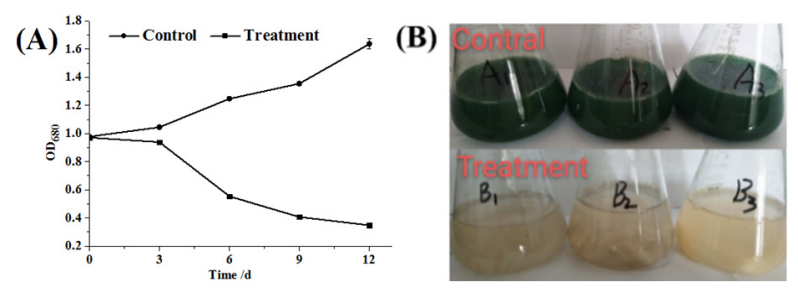

(C)
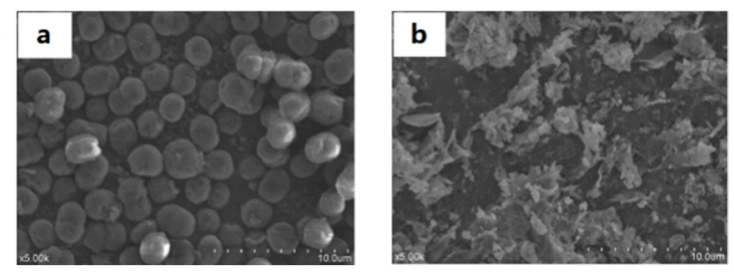

Fig. 1 Effect of chitosan fiber on M. aeruginosa ((A) Effect of chitosan fiber on the growth of $M$. aeruginosa (B) Color changes of $M$. aeruginosa (C) SEM of M. aeruginosa: (a) Control group, (b) Treatment group)

The concentration of algal cells in the blank group gradually increased, and the color gradually deepened until it turned dark green. Meanwhile, the treatment group became transparent after 12 days, and only a small amount of yellow precipitate remained. As shown in Figs $1 \mathrm{~B}$.

The microscopic morphology (SEM) of $M$. aeruginosa is shown in Figs. 1C (a)-(b). Fig. 1C shows that the normal cells of $\mathrm{M}$. aeruginosa are round and full. The appearance is smooth, the cells are close to a sphere status, and the individual is distinct. After 12 days of exposure to chitosan fibers, the algal cells were ruptured, internal matter was dissolved, and flocs were distributed on the surface and around the cells. This result may be due to the chitosan fibers directly or indirectly destroying 
the integrity of the cell membrane, thus inducing the internal solution of the cells to overflow.

\subsection{Inhibition effect on cell membrane permeability within $M$. aeruginosa}

The effect of chitosan fiber on non-electrolyte extravasation within $M$. aeruginosa is shown in Fig.2A.As shown in Fig. 2A, the content of non-electrolyte extravasation OD264 in the chitosan fiber group increased significantly with prolonged exposure time compared with that in the control group. The content of OD264 in the control group was relatively stable, whereas that in the treatment group reached the maximum at 1.61 times higher than the initial value. The amount of extravasation of non-electrolyte extravasation from day 6 to day 12 decreased slightly and stabilized afterward.
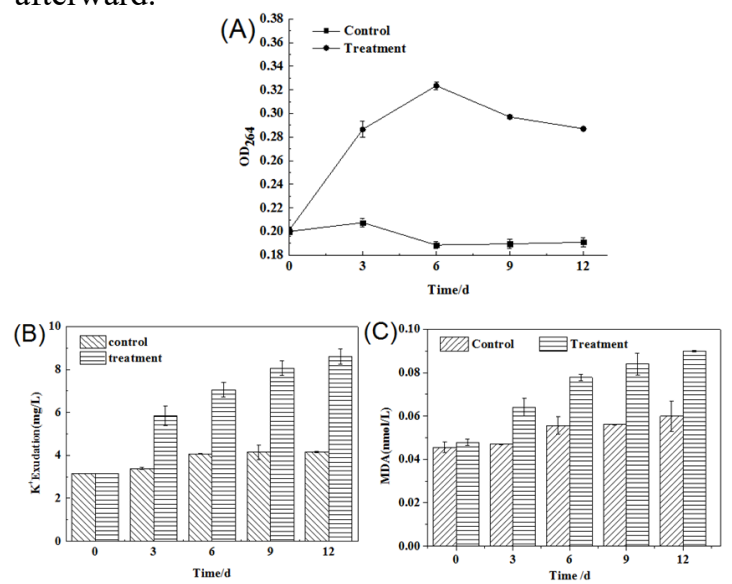

Fig.2 Effect of Chitosan Fiber on Cell Membrane Permeability ((A)Effect of chitosan fiber on $\mathrm{OD}_{264}$ of $M$. aeruginosa (B) Effect of chitosan fiber on intracellular $\mathrm{K}^{+}$released from $M$. aeruginosa $(\mathrm{C})$ Effect of chitosan fiber on MDA content within M. aeruginosa)

The effect of chitosan fiber on $\mathrm{K}+$ within $\mathrm{M}$. aeruginosa is shown in Fig. 2B.Fig. 2B shows that the $\mathrm{K}+$ content in the treatment group increased significantly with the exposure time compared with the control group. The $\mathrm{K}+$ content in the control group was relative stable, whereas that in the treatment group was 2.72 times than that of the control group at 12th day. After treatment with chitosan fiber, the cell membrane of $\mathrm{M}$. aeruginosa became selectively permeable, and the number of ions was exuded. When the algal cells were destroyed, the $\mathrm{K}+$ within the algal cells was completely released, and the mass concentration increased to $9.598 \mathrm{mg} / \mathrm{L}$. Finally, the cell membrane of $\mathrm{M}$. aeruginosa was severely damaged.

The effect of MDA content within $\mathrm{M}$. aeruginosa is shown in Fig. 2C.Fig. $2 \mathrm{C}$ shows that the MDA content in the chitosan fiber group increased significantly with exposure time compared with that in the control group. The MDA in the treatment group showed an upward trend with the concentration and increased from 0.0478 $\mathrm{nmol} / \mathrm{L}$ to $0.09 \mathrm{nmol} / \mathrm{L}$. Algal cell membranes are composed of unsaturated phospholipids and are sensitive to reactive oxygen species. On the third day, the concentration of MDA in algal cells of the treatment group was increased significantly, indicating that chitosan may cause severe oxidative stress on $\mathrm{M}$. aeruginosa and cause damage of cell membrane.

\subsection{Inhibition effect on Photosynthesis within $M$. aeruginosa}

The effects on the growth of $M$. aeruginosa can be revealed also by reduce of chlorophyll [18]. The effects of chitosan fibers on chlorophyll a and carotenoids are shown in Figs. 3(A) and (B), respectively.
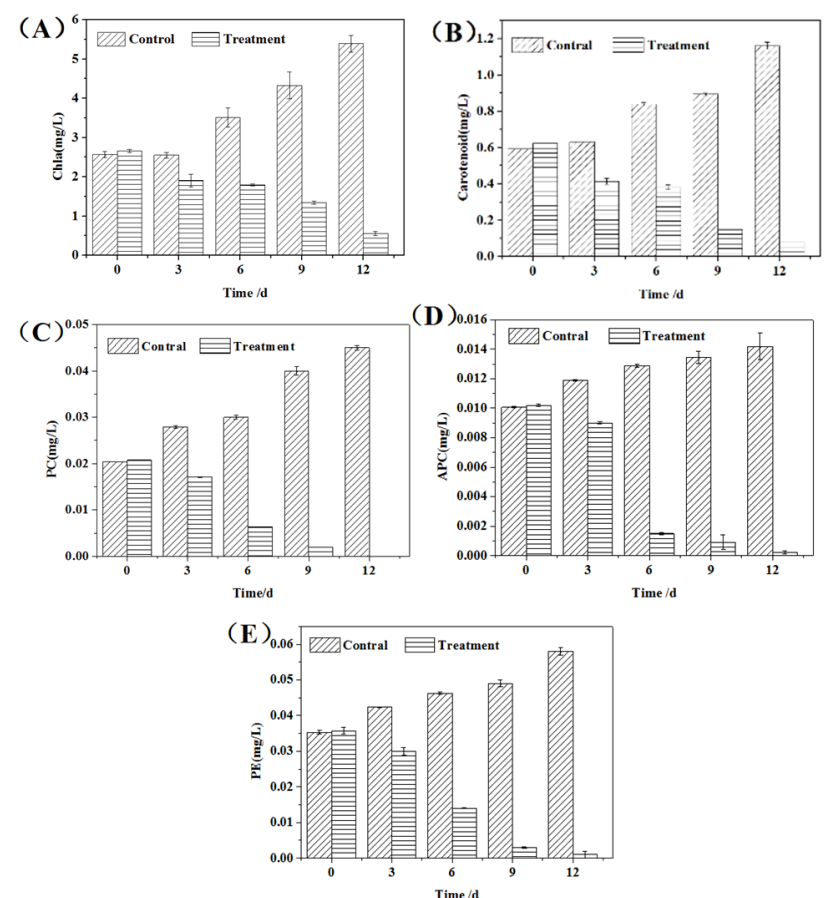

Fig. 3 Effect of chitosan fiber on photosynthesis of $M$. aeruginosa ((A) Chlorophyll a; (B) Carotenoids (C) PC, (D) APC, (E) PE)

Figs. 3 (A) and (B) show that chitosan fibers have a significant inhibitory effect on the concentration of chlorophyll a and carotenoids within $M$. aeruginosa compared with those in the control group. After 12 days of monitoring, the chlorophyll a of the control group increased from $2.66 \mathrm{mg} / \mathrm{L}$ to $5.39 \mathrm{mg} / \mathrm{L}$, whereas that of the treatment group showed a downward trend from 2.57 $\mathrm{mg} / \mathrm{L}$ to $0.55 \mathrm{mg} / \mathrm{L}$. Carotenoids showed the same trend. The carotenoid concentration increased from $0.592 \mathrm{mg} / \mathrm{L}$ to $1.16 \mathrm{mg} / \mathrm{L}$ in the treatment group but decreased from $0.623 \mathrm{mg} / \mathrm{L}$ to $0.079 \mathrm{mg} / \mathrm{L}$ in the treatment group. The IRs of chlorophyll a and carotenoids reached $89.6 \%$ and $93.1 \%$, respectively, on the 12 th day.

Fig. 3(C) shows that the chitosan fiber inhibited the expression of $\mathrm{PC}$ in $\mathrm{M}$. aeruginosa compared with the control group. The PC in the control group showed an upward trend from $0.0162 \mathrm{mg} / \mathrm{L}$ to $0.0318 \mathrm{mg} / \mathrm{L}$, whereas that in the treatment group showed a downward trend from $0.0171 \mathrm{mg} / \mathrm{L}$ to $0 \mathrm{mg} / \mathrm{L}$. The IR of PC reached $100 \%$ on the 12th day. Fig. 3(D) shows that the expression of APC within M. aeruginosa and the other algal protein in 
the control group showed an increasing trend from $0.0055 \mathrm{mg} / \mathrm{L}$ to $0.0177 \mathrm{mg} / \mathrm{L}$. Meanwhile, the other algal protein in the treatment group showed a downward trend from $0.0059 \mathrm{mg} / \mathrm{L}$ to $0.00 \mathrm{mg} / \mathrm{L}$. The IR of APC reached $100 \%$ on the 12 th day. Fig. 3(E) shows that the expression of $\mathrm{PE}$ in $\mathrm{M}$. aeruginosa was inhibited by the chitosan fiber. The PE in the control group showed an upward trend from $0.0341 \mathrm{mg} / \mathrm{L}$ to $0.0554 \mathrm{mg} / \mathrm{L}$, whereas that in the treatment group decreased from $0.0343 \mathrm{mg} / \mathrm{L}$ to $0.00 \mathrm{mg} / \mathrm{L}$. The inhibition effects of chitosan fiber for $\mathrm{PC}, \mathrm{APC}$, and PE are evident.

\subsection{Inhibition effect on soluble protein within $M$. aeruginosa}

The changes of soluble protein within $M$. aeruginosa are shown in Fig. 4.

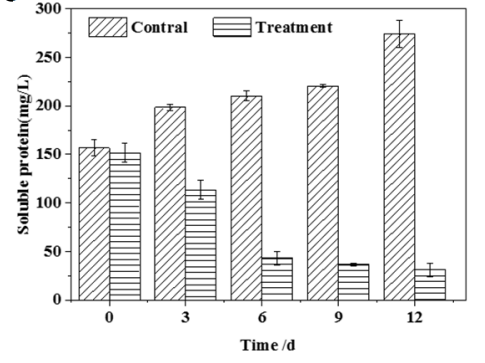

Fig. 4 Effect of chitosan fiber on soluble protein content within M. aeruginosa

Fig. 4 shows that chitosan fibers have a significant inhibitory effect on the synthesis of soluble proteins within M. aeruginosa. The soluble protein was increased from $156.8 \mathrm{mg} / \mathrm{L}$ to $274.4 \mathrm{mg} / \mathrm{L}$ in the control group and showed a downward trend from $151.4 \mathrm{mg} / \mathrm{L}$ to 31.27 $\mathrm{mg} / \mathrm{L}$ in the treated group. The protein content of cells was reduced, and the IR reached $88 \%$ on the 12 th day.

\subsection{Inhibition effect on SOD and CAT within $M$. aeruginosa}

As shown in Fig. 5(a), chitosan fiber significantly inhibited the activity of SOD within $M$. aeruginosa. SOD activity remained relatively stable in the control group, and the enzyme activity ranged from $0.0091 \mathrm{U} / 104$ cell to $0.0108 \mathrm{U} / 104$ cell. Meanwhile, the enzyme activity in the treatment group on the 12 th day decreased from 0.009 $\mathrm{U} / 104$ cell to $0.0035 \mathrm{U} / 104$ cell, and the IR reached $68 \%$.

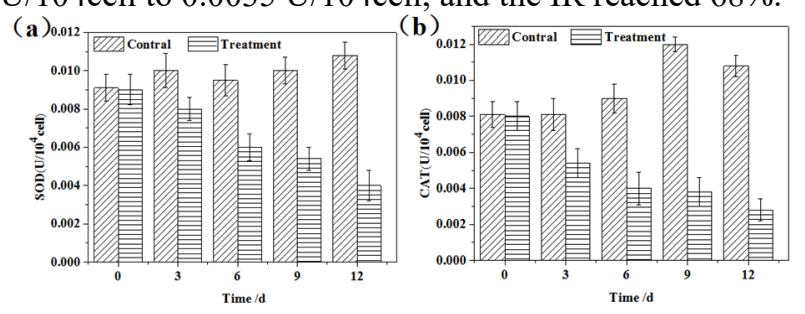

Fig. 5 Effect of chitosan fiber on enzyme activity within $M$. aeruginosa ((a) SOD; (b) CAT)

As shown in Fig. 5(b), chitosan fiber significantly inhibited the activity of CAT within M. aeruginosa. The
CAT activity in the control group remained relatively stable, and the enzyme activity increased from 0.0081 $\mathrm{U} / 104$ cell to $0.0108 \mathrm{U} / 104$ cell. Meanwhile, the activity of CAT in the treatment group on the 12th day decreased from $0.008 \mathrm{U} / 104$ cell to $0.0028 \mathrm{U} / 104$ cell, and the IR reached $74 \%$.

\subsection{Inhibition effect on the microcystin}

When algal cells are destroyed, algal toxins may be released, especially toxins produced by $M$. aeruginosa [19-20]. Fig. 6 shows that chitosan fibers have an inhibitory effect on the release of algal toxins from $\mathrm{M}$. aeruginosa compared with the control group. The concentration of algal toxins within $M$. aeruginosa in the chitosan fiber group tends to decrease. The microcystin content in the control group was increased from 0.22 $\mathrm{mg} / \mathrm{L}$ to $1.28 \mathrm{mg} / \mathrm{L}$. The initial value of microcystins in the treatment group was approximately $0.24 \mathrm{mg} / \mathrm{L}$. The value was below the detection limit on the 6th day. The IR of chitosan fiber to the microcystin reached $100 \%$. During the whole experiment, the content of microcystins in the treated samples was much lower than that of the control group.

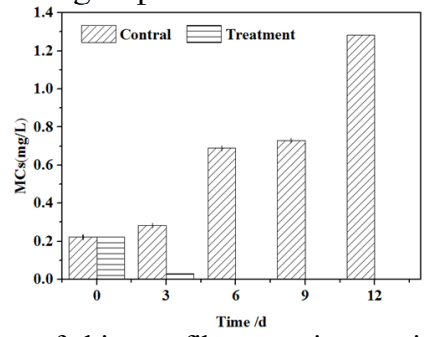

Fig. 6 Effect of chitosan fiber on microcystin within $M$. aeruginosa

Microcystins are found in cyanobacterial cells, synthesized intracellularly, and released into the water after death [21]. The production of microcystins in the experimental group was significantly lower than that in the control group throughout the experiment. This result indicates that microcystins may be decomposed or absorbed by chitosan fibers.

\section{Conclusion}

This study indicated that chitosan fiber could suppress the cell proliferation of $M$. aeruginosa. Chitosan fiber reduced $M$. aeruginosa photosynthetic abilities by triggering the downregulation of genes involved in Chl synthesis and phycobiliprotein synthesis, which affected the cell growth. After treatment by chitosan fiber, the protein content decreased, MDA content increased, and the algal cells were destroyed. Therefore, chitosan fiber can be used to inhibit M. aeruginosa growth.

\section{Acknowledgments}

This work was supported by the Foundation (No. 18YFJLCG00120) of Tianjin science and technology project (Tianjin Municipal Science and Technology 
Bureau), and (No. 201808) of Tianjin Key Laboratory of Marine Resources and Chemistry (Tianjin University of Science \& Technology), P. R. China

\section{References}

1. M. Xiao, M. Li, \& C. S. Reynolds, Colony formation in the cyanobacterium microcystis. Biological Reviews, 93(3). (2018)

2. Y. Oyama, B. Matsushita, \& T. Fukushima, Distinguishing surface cyanobacterial blooms and aquatic macrophytes using landsat/tm and etm+ shortwave infrared bands. Remote Sensing of Environment, 157, 35-47. (2015)

3. C. A. Weirich, \& T. R. Miller, Freshwater harmful algal blooms: toxins and children $\backslash$ "s health. Current Problems in Pediatric and Adolescent Health Care, 44(1), 2-24. (2014)

4. P. W. Perschbacher, \& G. M. Ludwig, Effects of diuron and other aerially applied cotton herbicides and defoliants on the plankton communities of aquaculture ponds. Aquaculture, 233(1-4), 197-203. (2003)

5. E. Costas, \& V. Lopez-Rodas, Copper sulphate and DCMU-herbicide treatments increase asymmetry between sister cells in the toxic cyanobacteria Microcystis aeruginosa: Implications for detecting environmental stress. Water research, 40(12), 2447-2451. (2006)

6. D. Jančula, \& B. Maršálek, Critical review of actually available chemical compounds for prevention and management of cyanobacterial blooms. Chemosphere, 85(9), 1415-1422. (2011)

7. C. Zhang, Y. L. Yi, K. Hao, G. L. Liu, \& G. X. Wang, Algicidal activity of Salvia miltiorrhiza Bung on Microcystis aeruginosa-Towards identification of algicidal substance and determination of inhibition mechanism. Chemosphere, 93(6), 997-1004. (2013)

8. S. Nakai, Y. Inoue, M. Hosomi, \& A. Murakami, Myriophyllum spicatum-released allelopathic polyphenols inhibiting growth of blue-green algae Microcystis aeruginosa. Water Research, 34(11), 3026-3032. (2000)

9. Y. Hong, H.Y. Hu, X. Xie, A. Sakoda, M. Sagehashi, F.M. Li, Gramine-induced growth inhibition, oxidative damage and antioxidant responses in freshwater cyanobacterium Microcystis aeruginosa. Aquat. Toxicol. 91, 262-269. (2008)

10. M. Prabaharan, Bioactivity of Chitosan Derivative. Springer International Publishing. (2014)

11. M. Dumont, R. Villet, M. Guirand, A. Montembault,
T. Delair, S. Lack, M. Barikosky, A. Crepet, P. Alcouffe, F. Laurent, L. David, Processing and antibacterial properties of chitosan-coated alginate fibers. Carbohydrate Polymers, 190, 31-42. (2018)

12. S. H. Duan, M. Y. Zhang, X. L. Cao, J. G. Cao, Z. Z. Yang, \& H. L. Liu, Study on Allelopathic Inhibition of Chinese Herbal Extracts on Microcystis aeruginosa. Water Supply and Drainage, (4), 14. (2018)

13. Z. Wang, D. Li, G. Li, Y. Liu, Mechanism of photosynthetic response in Microcystis aeruginosa PCC7806 to low inorganic phosphorus. Harmful Algae, 9(6):613-619. (2010)

14. M. P. Padgett, D. W. Krogmann, Large scale preparation of pure phycobiliproteins. Photosynthesis Research, 11(3):225-235. (1987)

15. M. M. Bradford, A rapid and sensitive method for the quantitation of protein utilizing the principle of protein-dye binding. Academic Press, 72(s 12):248-254. (1976)

16. X. Hou, J. Huang, J. Tang, N. Wang, L. Zhang, L. $\mathrm{Gu}$, Y. Sun, Z. Yang, \& Y. Huang, Allelopathic inhibition of juglone (5-hydroxy-1, 4-naphthoquinone) on the growth and physiological performance in Microcystis aeruginosa. Journal of environmental management, 232, 382-386. (2019)

17. C. T. Shiu, T. M. Lee, Ultraviolet-B-induced oxidative stress and responses of the ascorbateglutathione cycle in a marine macroalga Ulva fasciata. Journal of Experimental Botany. 56, 28512865. (2005)

18. P. Meng, H. Pei, W. Hu, Z. Liu, X. Li, \& H. Xu, Allelopathic effects of Ailanthus altissima extracts on Microcystis aeruginosa growth, physiological changes and microcystins release. Chemosphere, 141, 219-226. (2015)

19. Y. Wu, P. G. Kerr, Z. Hu, \& L. Yang, Removal of cyanobacterial bloom from a biopond-wetland system and the associated response of zoobenthic diversity. Bioresource technology, 101(11), 3903-3908. (2009)

20. H. Y. Pei, C. X. Ma, W. R. Hu, \& F. Sun, The behaviors of Microcystis aeruginosa cells and extracellular microcystins during chitosan flocculation and flocs storage processes. Bioresource technology, 151, 314-322. (2014)

21. D. R. D. Figueiredo, U. M. Azeiteiro, S. M. Esteves, F. J. M. Gonçalves, \& M. J. Pereira, Microcystin-producing blooms-a serious global public health issue. Ecotoxicology \& Environmental Safety. 59(2), 151-163. (2004) 